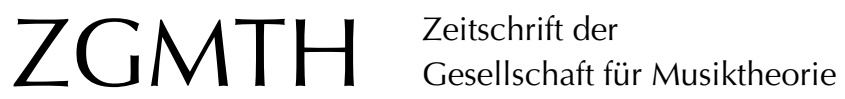

Popovic, Tihomir (2011): »Leaving the key« in "gravity and piety«. Zur Tonartbehandlung in William Byrds Fantasien für Tasteninstrumente. ZGMTH 8/3, 407-426. https://doi.org/10.31751/651

(C) 2011 Tihomir Popovic

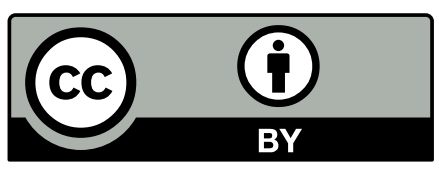

Dieser Text erscheint im Open Access und ist lizenziert unter einer Creative Commons Namensnennung 4.0 International Lizenz.

This is an open access article licensed under a Creative Commons Attribution 4.0 International License.

veröffentlicht / first published: 29/03/2012

zuletzt geändert / last updated: 09/11/2013 


\section{»Leaving the key« in "gravity and piety« Zur Tonartbehandlung in William Byrds Fantasien für Tasteninstrumente}

Tihomir Popovic

ABSTRACT: Eine quellenorientierte Untersuchung der Modusbehandlung in der Instrumentalmusik William Byrds gehört zu den Desideraten der Musikforschung. Der vorliegende Beitrag untersucht die Tonartendispositionen der für Tasteninstrumente geschriebenen Fantasien des bedeutendsten elisabethanischen Komponisten vor dem Hintergrund der zeitgenössischen - insbesondere englischen - Musiktheorie. Zum Vergleich werden die Fantasien anderer englischer Komponisten der Epoche herangezogen. Schließlich werden die Analyseergebnisse auch im Kontext der englischen Sozial- und Kulturgeschichte interpretiert.

Obwohl Byrd zeitgenössischen Zeugnissen zufolge als der bedeutendste Komponist der elisabethanischen Epoche erachtet wurde ${ }^{1}$ und die Fantasie als Gattung der Instrumentalmusik von höchstem kompositorischem Rang galt, liegen bisher kaum tonartanalytische Betrachtungen der Fantasien William Byrds vor: So wird etwa in Oliver Neighbours Standardwerk ${ }^{2}$ über Byrds Tastenmusik die Tonartbehandlung kaum angesprochen, während die zentralen modusanalytischen Studien ${ }^{3}$ die Instrumentalmusik Byrds bestenfalls am Rande thematisieren. Auch in Bernhard Meiers Studie über die Modusbehandlung in der Instrumentalmusik des 16. Jahrhunderts ${ }^{4}$ wird nur ein sehr kurzes, für Byrds Gesamtschaffen kaum repräsentatives Präludium erwähnt. ${ }^{5}$ Die bisher einzige monographische Studie über Byrds Modusbehandlung, die John Harley vorgelegt hat ${ }^{6}$, behandelt hauptsächlich vokale Kompositionen.

Der vorliegende Beitrag geht diesem Forschungsdesiderat nach und untersucht die Modusbehandlung in William Byrds Fantasien für Tasteninstrumente ausgehend von den zeitgenössischen Moduslehren und unter besonderer Berücksichtigung der englischen

1 Vgl. etwa die Äußerungen Thomas Morleys (1597/1937, Widmung, o. S.), John Baldwins (zitiert u.a. in Boyd 1962, 311, und Harley 1997, 367) sowie Thomas Peachams (1962, 112); zur zeitgenössischen Byrd-Rezeption vgl. insbesondere Harley 1997, 363-368.

2 Neighbour 1978.

3 Eine Auswahl dieser Studien wird in Anm. 39 angeführt.

4 Meier 1992a.

5 Ebd, 121.

6 Harley 2005. 
Musiktheorie der Epoche. Dabei soll die Tonartbehandlung in Byrds Musik nicht nur beschrieben, sondern auch sozial- und kulturhistorisch kontextualisiert und interpretiert werden.

\section{Die Instrumentalfantasie des 16. Jahrhunderts: eine `Gattung׳?}

In den Standardwerken der Tastenmusikforschung - etwa bei Willy Apel', Oliver Neighbour $^{8}$ und Arnfried Edler ${ }^{9}$ - bezeichnet der Begriff `Fantasie`, sofern er auf Musik des 16. und 17. Jahrhunderts bezogen wird, frei konzipierte, sprosanah $\wedge^{10}$ aufgebaute Kompositionen, die, insbesondere in den Exordialabschnitten, durch eine intensive Verwendung der Imitationspolyphonie geprägt sind. ${ }^{11}$ Die im England des 16. Jahrhunderts für solche Kompositionen üblichen Begriffe waren insbesondere 'Fancy`, 'Voluntary 'Fantasia.${ }^{12}$ Martin Klotz hat darüber hinaus vorgeschlagen, Fantasien mit hexachordalen Soggetti als eine eigene ‘Gattung`der Musik für Tasteninstrumente zu betrachten. ${ }^{13}$ Doch wirkt sein Argument, `Hexachordfantasien s seien in den Quellen nicht als Fantasien bezeichnet worden ${ }^{14}$, angesichts der allgemeinen Flexibilität der Terminologie im 16. Jahrhundert wenig überzeugend. So trugen etwa Grounds - Variationen auf präexistente oder eigens komponierte Bassvorlagen -, denen eine bekannte Vorlage zugrunde lag, deren jeweiligen Titel (z. B. Byrds Hunt's up MB XXVII/40 oder Chi passa MB XXVII/1915); nur wenn keine bekannte Vorlage verwendet wurde, kamen die allgemein üblichen Gattungsbegriffe zur Geltung (z. B. Byrds My Lady Nevells Ground MB XXVIII/61). Ebenso wurden auch Fantasien, denen eine besondere Vorlage - etwa die Töne des Hexachords - zugrunde lag, nach ihrer Vorlage benannt (z. B. Ut, re, mi MB XXVIII/64 oder Ut, mi, re MB XXVIII/65 von William Byrd), während ansonsten die Gattungsbegriffe ıVoluntary` oder 'Fancy< gebräuchlich waren. Auch vor dem Hintergrund, dass statische und eng umrissene `Gattungsbegriffe` generell als problematisch gelten dürften ${ }^{16}$, scheint die Postulierung eines separaten Gattungsbegriffes im Falle der Hexachordfantasie verzichtbar.

\section{Die Fantasie im kulturgeschichtlichen Kontext}

Thomas Morley, Schüler William Byrds und der einflussreichste englische Theoretiker der Epoche, bezeichnete die Instrumentalfantasie als »The most principall and chiefest

7 Apel 1972.

8 Neighbour 1978.

9 Edler 1997.

10 Zur Bedeutung des musikalischen Prosabegriffs, insbesondere in Bezug auf spätere Epochen s. insbesondere Danuser 1975; in Bezug auf den Prosacharakter der Renaissance-Polyphonie s. ebd., 11. Zur Begriffsbestimmung s. Danuser 1997.

11 Vgl. Apel 1972, 202-206, Neighbour 1978, 221-258 sowie Edler 1997, 354-359.

12 Ebd.; vgl. insbesondere das zitierte Werk Neighbours.

13 Klotz 2005, 267.

14 Ebd.

15 Vgl. das Verzeichnis der musikalischen Quellen. 
kind of musicke which is made without a dittie «..$^{17}$ Diese Vorrangstellung mag darin gründen, dass die Fantasie durch die Verwendung der Imitationspolyphonie und die freie, sprosanahe، Aufbauart den Gattungen der Vokalmusik, vor allem der Motette, besonders nahe steht und - im Unterschied zu den meisten anderen Gattungen der Musik für Tasteninstrumente - keinen systemischen Bezug zur Tanz- und Volksmusik erkennen lässt. ${ }^{18}$ Die Gesamtgestaltung und die Satztechnik mancher Fantasien - etwa der Voluntary for My Lady Nevell William Byrds - weisen eine so weitreichende Verwandtschaft mit der Vokalmusik auf, dass man zuweilen den Eindruck gewinnen kann, es handle sich um Intavolierungen von Motetten.

Die Entstehung der Gattung `Fantasie` ist von den gesellschaftlichen Umbrüchen der Tudor-Epoche nicht zu trennen. Arnfried Edler verweist in diesem Zusammenhang auf Auswirkungen von Kirchenreformen auf die Musik für Tasteninstrumente. ${ }^{19}$ Die puritanischen Bewegungen führten im Tudor-Königreich vielfach zu einer radikalen Reduzierung und Simplifizierung der Kirchenmusik bis hin zum zeitweiligen Verbot des liturgischen Orgelspiels in englischen Kirchen. ${ }^{20}$ Möglicherweise begünstigte diese Entwicklung die Entfaltung einer vornehmlich polyphonen, aber säkularen Musikgattung für Tasteninstrumente. Aufschlussreich ist in diesem Zusammenhang die zunehmende curriculare Präsenz der Instrumentalmusik an englischen Universitäten: Die Musik der geistlichen Eliten, deren gesellschaftlicher Einfluss im England des 16. Jahrhunderts zunehmend schwand, wurde in säkularisierter Form zum skulturellen Kapital ${ }^{21}$ der neuen, aufgestiegenen Bildungseliten des Tudor-Zeitalters.

Eine Analyse der musikalischen Widmungspraxis William Byrds, John Bulls und Orlando Gibbons', die von ihren Zeitgenossen als die „Triumvirn der Musik« glorifiziert wurden $^{22}$, stützt diese These. ${ }^{23}$ Nur eine Fantasie dieser Komponisten - die bereits erwähnte Voluntary for My Ladye Nevell von William Byrd - trägt eine Widmung; in den überlieferten Werken Thomas Tompkins' und Peter Philips' ist keine Fantasie mit einer Widmung nachweisbar. ${ }^{24}$ Dagegen sind in Kompositionen anderer Gattungen - insbesondere bei den stilisierten Hoftänzen Pavane und Galliarde - Widmungen, vornehmlich an Adelsangehörige, zahlreich. ${ }^{25}$ Es ist womöglich kein Zufall, dass die einzige sWid-

16 Vgl. hierzu etwa Danuser 1995.

17 Morley 1597/1937, 181.

18 Aus diesem Grund werden im Rahmen des vorliegenden Beitrags auch historische Musiktermini verwendet, die in Bezug auf Motetten angemessen wären.

19 Vgl. Edler 1997, 354.

20 Vgl. ebd.

21 Dieser Begriff wird hier im Sinne Pierre Bourdieus verwendet (ausgeführt insbesondere in Bourdieu 1992).

22 Die Bezeichnung "triumviri of Musicke» in Bezug auf Byrd, Bull und Gibbons stammt aus den Vorwort-Gedichten der Sammlung Parthenia (vgl. das Verzeichnis der musikalischen Quellen).

23 Die Analyse der Widmungspraxis basiert auf MB XXVII und XXVIII (für Byrd), MB XIV und XIX (für Bull) sowie MB XX (für Gibbons).

24 Untersucht wurden die Quellen MB V (für Tomkins) sowie MB LXXVII (für Philips).

25 Ausführlicher erörtert wird diese Widmungspraxis in der Dissertation des Verfassers (Popovic 2010, Teil 5). 
mungsfantasie` der genannten englischen Komponisten keinem Nachfahren einer alten adligen Familie, sondern Elizabeth Bacon-Neville gewidmet ist ${ }^{26}$, einer Tochter des durch Bildung sozial aufgestiegenen Großsiegelbewahrers Sir Nicholas Bacon ${ }^{27}$, in dessen Familie auch in die Bildung weiblicher Nachkommen investiert wurde. ${ }^{28}$ Byrds Widmungsträgerin `Ladye Nevelk war zudem die Halbschwester eines der bedeutendsten Philosophen und Universalgelehrten der Epoche: Francis Bacon, Baron Verulam, Viscount St. Alban. ${ }^{29}$ Die englische Fantasie des ausgehenden 16. Jahrhunderts ist demnach sowohl hinsichtlich ihrer musikalischen Gestaltungsprinzipien als auch im Hinblick auf ihren gesellschaftlichen Kontext eng verbunden mit dem Aufstieg der neuen, humanistischen Bildungselite des Tudor-Zeitalters.

\section{Zum Fantasiebegriff Thomas Morleys}

Thomas Morley begründete sein oben zitiertes Werturteil über die Fantasie mit dem freien Gesamtkonzept dieser Gattung, die höchstmögliche künstlerische Entfaltung ermögliche. Er beschrieb das Komponieren einer Fantasie folgendermaßen:

When a musician taketh a point [in diesem Kontext: Soggetto] at his pleasure, and wresteth and turneth it as he list [likes], making either much or little of it according as shell seeme best in his own conceit. In this may more art be showne then in any other musicke, because the composer is tide to nothing but that he may adde, deminish, and alter at his pleasure. ${ }^{30}$

Morleys weiteren Ausführungen zufolge erstreckt sich diese Freiheit auf die formale Gliederung und die Satztechnik, nicht aber auf die Tonartbehandlung:

And this kind will beare any allowances whatsoeuer tolerable in other musick, except changing the ayre \& leauing the key, which in fantasie may neuer bee suffered. ${ }^{31}$

Die Formulierungen »changing the ayre« und »leaving the key« verweisen, wie an anderer Stelle deutlich wird, auf den Wechsel der durch den Melodieverlauf (sairs) und die finalisdefinierte Modusbasis (`key«) determinierten Tonart. ${ }^{32}$ Dieses `Tonartwechselverbot`

26 Die genaue Identität Lady Nevilles wurde von Christopher Foley und John Harley entdeckt (vgl. Foley 2005 sowie Harley 2005a) und vom Verfasser durch zusätzliche Argumente bestätigt (vgl. Popovic 2010, Kapitel 2.1 und 2.2).

27 Zu Nicholas Bacon vgl. etwa Titler 1976 und 2004.

28 Vgl. etwa einen Brief Sir Nicholas' an seinen Sohn Nicholas, zitiert in: Smith 1972, 93.

29 Vgl. dazu exemplarisch neuere Beiträge wie Solomon/Martin 2005 und Desroches 2006.

30 Morley 1597/1937, 181; Morleys Ausführungen scheinen auch Praetorius beeinflusst zu haben; vgl. Praetorius 1619/1978, 21.

31 Morley 1597/1937, 181.

32 Ebd., 147. Zu dieser Terminologie Morleys s. auch Hauge 1997, 105; zur Bedeutung der Termini sair und saries allgemein s. etwa Palisca 1994, 346-363 sowie Palisca 1985, 376-378. Vgl. auch Zimmerman 1980. 
wird im Anhang der Abhandlung relativiert: Dort erlaubt Morley die Verwendung zweier Modi innerhalb einer Komposition, wenn der eine von ihnen plagal und der andere authentisch sei. Gemeint sind offenkundig zwei Modi gleicher Finalis (etwa Dorisch und Hypodorisch $^{33}$ ) - eine Annahme, die durch die gegebenen Beispiele bestätigt wird. Die betreffende Stelle könnte demnach so verstanden werden, dass der Autor die Mixtio tonorum - den zeitweiligen Wechsel in die Tonart mit gleicher Finalis und unterschiedlichem Ambitus - erlauben, die Commixtio tonorum hingegen - den Wechsel in eine Tonart mit anderer Finalis - verbieten wollte. ${ }^{34}$

\section{Modale Individualkonzepte Byrds: drei Fallbeispiele ${ }^{35}$}

Der erwähnte Traktat ist Morleys Lehrer William Byrd gewidmet und damit zugleich die einzige musiktheoretische Quelle, die mit Sicherheit im Umkreis Byrds entstanden ist. Angesichts des Moduswechselverbots im Hauptteil des Traktats überrascht das markanteste Charakteristikum der Byrdschen Fantasien: Sie basieren nahezu durchgehend auf Moduswechseln. Exemplarisch werden hier drei Fälle, die jeweils unterschiedliche Konzepte der Tonartbehandlung aufweisen, vorgestellt.

Die Exordialmelodik der Hexachord-Fantasie Ut, re, mi (MB XXVIII/64) weist eindeutige Charakteristika des Hypomixolydischen auf: Die Voces regales, Diskant und Tenor, bewegen sich im hypomixolydischen Bereich, die beiden sdienenden Stimmen, Alt und Bass, sind mixolydischen Charakters. Auch die Klauselbildung des exordialen Abschnitts (T. 1-23) entspricht der modalen Tradition ${ }^{36}$ : Die erste Klausel findet auf $c$ (T. 6), der alten hypomixolydischen Repercussa, statt, alle weiteren Klauseln auf der Finalis oder der Oberquinte.

Der weitere Verlauf der Fantasie ist dagegen keineswegs so konventionell. Obwohl die Melodiebildung und die Klauseldisposition wiederholt das (Hypo-)Mixolydische zu bestätigen scheinen, können manche Abschnitte nur als Übergang in einen anderen Modus gedeutet werden. Ab Takt 30 ist die Melodik hypolydischen Charakters (vgl. Beispiel 1). In Takt 37 erweitert sich der Ambitus des den $>$ Point ${ }^{37}$ vortragenden Diskants auf den Bereich des Lydischen und erreicht das $f^{2}$. Die Ecktöne des Gesamtambitus sind

33 Es werden im vorliegenden Beitrag übersichtshalber die spseudoantiken ^ Namen der Modi bevorzugt.

34 Hier wird die skontinental-europäische` zeitgenössische Terminologie verwendet, wie etwa von Meier (1974 und 1992a) sowie Dahlhaus (2001a), da Morley selbst keine Termini für die beschriebenen Sachverhalte geprägt hat.

35 Der vorliegende Abschnitt basiert auf dem Anhang zu Teil 5 der Dissertation des Autors (Popovic 2010).

36 Soweit sich der vorliegende Beitrag auf die Konventionen der smodalen Tradition ‘ beruft, knüpft er an die Studien Meiers (1974, 1977, 1992a, 1992b), Powers' (1981, 1989, 1992a, 1992b, 1998) und Dahlhaus' (insbesondere 2001a und 2001b) an, wobei die Untersuchungen Meiers eine besondere Position einnehmen, da sich Meier auch mit der Modalität der Instrumentalmusik des 16. Jahrhunderts intensiv auseinander gesetzt hat (1977 und 1992a). Für eine kritische Diskussion der Forschungsansätze von Meier, Powers und Dahlhaus vgl. Popovic 2010 sowie die Habilitationsschrift Wolfgang Horns (2005).

37 Zeitgenössischer englischer Terminus für Soggetto (vgl. etwa Morley 1597/1937, 181). 
nun $F$ und $f^{2}$; der Point setzt in allen Stimmen entweder auf $c$ oder auf $f$ ein, wie es im Hypolydischen und Lydischen der Regelfall ist. Dementsprechend stehen auch die Klauseln des gesamten hypolydisch-lydischen Abschnittes hauptsächlich auf $f$ (T. 32, 35, 40, 41, 50; vgl. Beispiel 1) und auf c (T. 39, 41), der sekundären Klauselstufe des Lydischen.
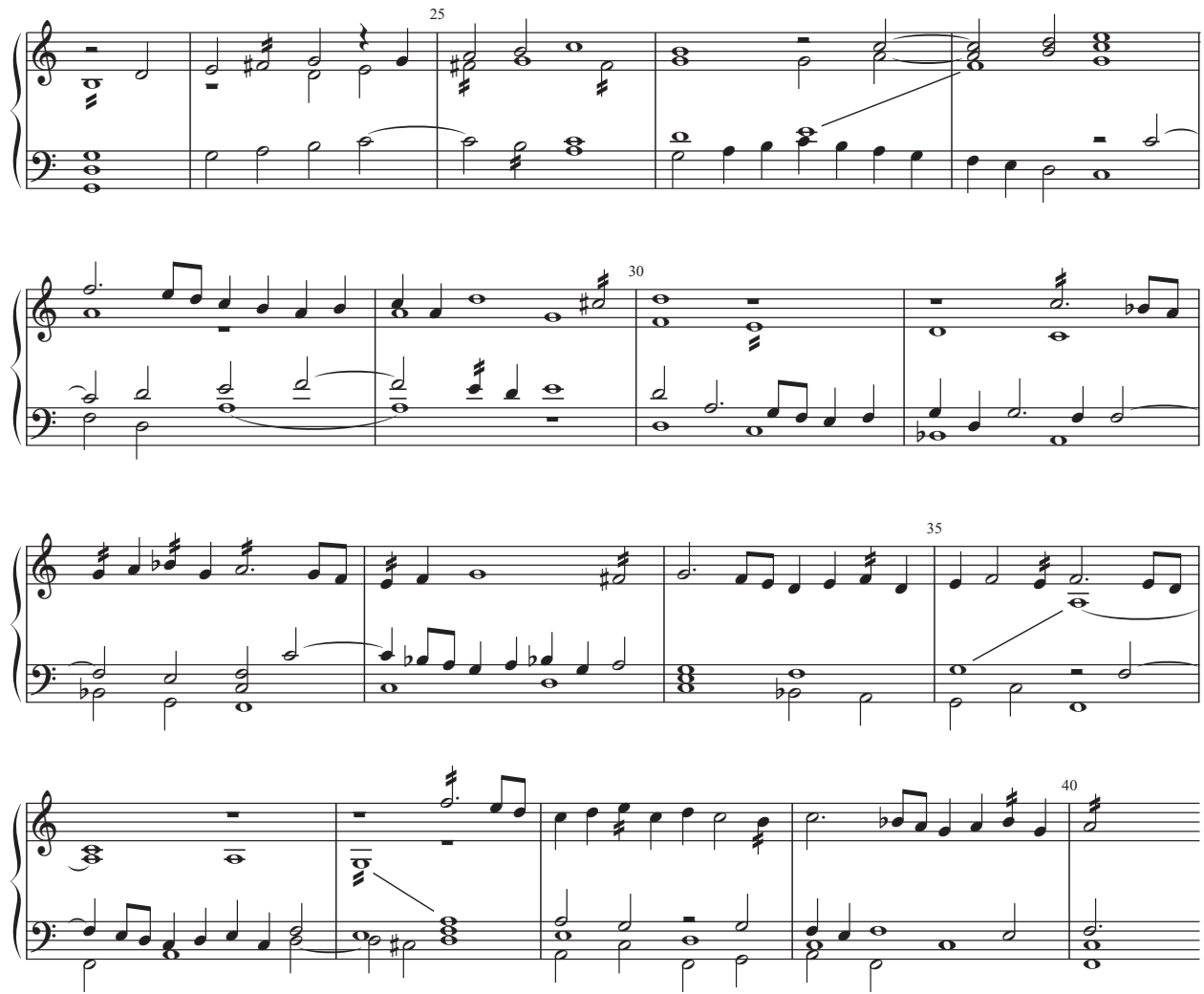

Beispiel 1: William Byrd, Ut, re, mi, MB XXVIII/64, T. 23-40

Der Übergang vom Hypomixolydischen zum Lydischen (T. 26-31, vgl. Beispiel 1) verläuft über einen Abschnitt, dessen Diskant hypodorische Züge trägt: Seine Untergrenze ist das $a^{1}$ - die Untergrenze des hypodorischen Ambitus in der Diskantlage -, während das $f^{2}$, die traditionelle hypodorische Repercussa, die markante Obergrenze (T. 28) und das $d^{2}$ die Klauselstufe (T. 30) bilden. Das $d$ ist zwar eine traditionelle Klauselebene auch des Hypomixolydischen, doch sprechen die genannten melodischen Merkmale gegen eine entsprechende Zuordnung.

Ab Takt 54 weist der Satz wieder hypomixolydische Charakteristika auf. Die in Takt 66 einsetzende Point-Durchführung verlagert den Ambitus in den Bereich des Mixolydischen: Der den Point vortragende Diskant bewegt sich nun hauptsächlich zwischen $g^{1}$ und fis $^{2}$. Das Zentrum der Melodie- und Klauselbildung wird jedoch bald das $d$ 
(T. 68 ff.). Die Melodik dieses Abschnittes ist eindeutig plagal, der Oberterz-Charakter dural (fis ist durchwegs präsent).

Die in Takt 75 beginnende Pars der Fantasie trägt Eigenschaften des zweifach transponierten Hypodorischen. Die Clausula primaria des Abschnittes steht auf $c$ (mit kleiner Terz), die Secundaria auf g. Insofern findet in diesem Abschnitt nicht nur ein Modus-, sondern auch ein Systemwechsel vom Cantus durus hin zum Cantus fictus (2 b) statt. ${ }^{38}$

Es folgt ein modal schwer bestimmbarer Abschnitt (T. 82 ff.): Die Bewegung des Diskants, der den Point vorträgt, spräche für Jonisch, die Klauseldisposition und die Melodieführung der anderen Stimmen lassen zunächst auf eine Tonart mit der Finalis $f$, dann auf eine mit Finalis $d$ schließen. Ab Takt 97 weist der Satz wieder hypomixolydische Charakteristika auf, wobei in Takt 101 eine Clausula peregrina auf e festzustellen ist. Die Melodik des darauf folgenden Abschnitts (T. 107 ff.) trägt anfangs Merkmale des Hypomixolydischen, weitet sich später (T. $115 \mathrm{ff}$.) zum authentischen, mixolydischen Ambitus und kehrt schließlich in den plagalen Rahmen zurück (T. 188f.). Dabei ist die Klauseldisposition dieses Abschnitts modal uncharakteristisch, insofern Klauseln auf a und e (T. 114, 116) stehen.

Der darauf folgende, virtuose Abschnitt (T. $125 \mathrm{ff}$.) bewegt sich in der ursprünglichen Tonart. Die abschließende Pars (T. 146 ff.) weist hinsichtlich der Melodik hypomixolydischen Charakter auf. Auch dieser Abschnitt beinhaltet eine Klausel auf der Obersekunde a (T. 153).

Aus der Durchführung des Hexachord-Soggettos auf verschiedenen Tonebenen resultieren in Ut, re, mi große Freiheiten in der Modusbehandlung. Es ist hier offensichtlich Teil des modalen Konzepts, auf allen Hexachordstufen zu klausulieren und dadurch dem Titel der Komposition auch über die Einsatzebenen des Soggettos hinaus Rechnung zu tragen. Andererseits weisen das modal strenge Exordium der Komposition und die Rückkehr in das Hypomixolydische an ihrem Ende auf eine bewusste Verwendung der modalen Technik hin, die in den weniger exponierten Abschnitten unkonventioneller verwendet wird.

Ganz anders ist die modale Disposition der Voluntary MB XXVII/27. ${ }^{39}$ Ihr Exordium ist äolisch geprägt, der weitere Verlauf dagegen hypojonisch. An der Übergangsstelle aus dem ersten in den zweiten Modus der Komposition schließen Tenor und Bass mit Klauseln auf $A / a$, während der Diskant bereits einen neuen Point im neuen Modus vorträgt (T. 7, vgl. Beispiel 2): Letzterer beginnt auf der Oberquint $g^{2}$, die erste Klausel wird auf $C^{2}$ gebildet (T. 9). Bemerkenswert ist, dass der neue Point aus dem Material des ersteren, äolischen abgeleitet zu sein scheint: Beide sind skalar angelegt, was bei den späten, glareanschen Modi auch keinen Sonderfall darstellen dürfte. Der Diskant bewegt sich nach dem Moduswechsel zwischen $g^{1}$ und $g^{2}$, was den hypojonischen Modus bestätigt; auch der Bass ist eindeutig hypojonisch. Die Mittelstimme steht im Moduspaar JonischHypojonisch und erstreckt sich über die Umfänge der beiden Modi.

38 Zur Terminologie s. etwa Dahlhaus 2001a, 158. Der englische Modustheoretiker Charles Butler nennt den Cantus fictus »scala mollaris« $(1636 / 1970,86)$.

39 Hier wird die kürzere Version dieser Fantasie (überliefert in My Ladye Nevells Booke, Nr. 42, (Beispiel 2) berücksichtigt, da dies die Besprechung übersichtlicher macht, die modale Gestaltung aber nicht berührt. In der Ausgabe MB XXVII/27 beginnt diese kürzere Version in Takt 47. 

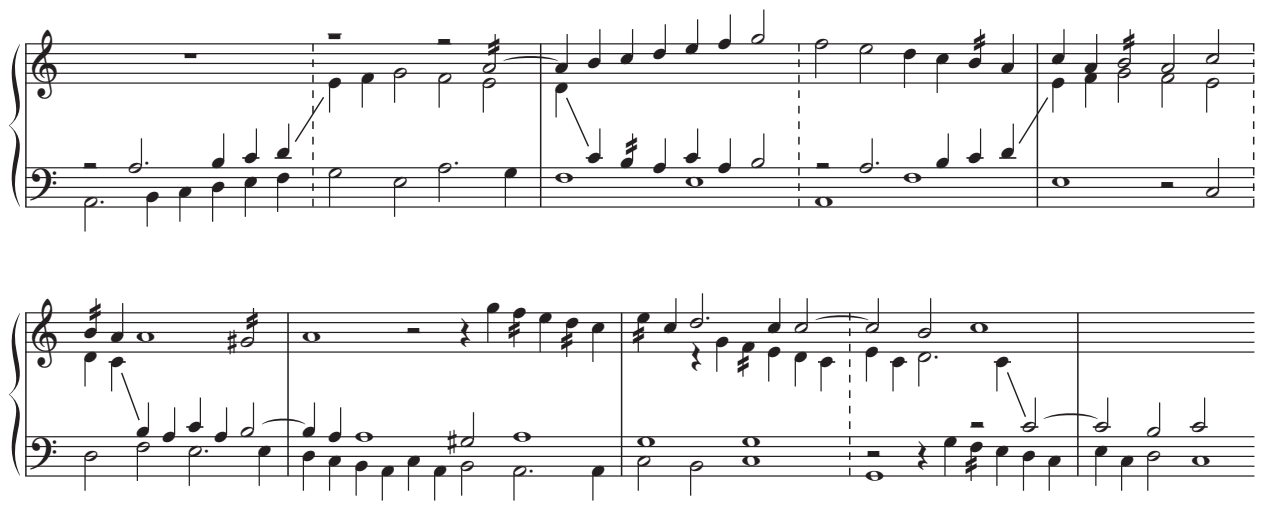

Beispiel 2: William Byrd, My Ladye Nevells Booke, Nr. 42, Beginn (vgl. MB XXVVII/27, T. 47 ff.)

Bernhard Meier hätte die Komposition womöglich zu den `Werken mit modal uneinheitlichem Verlauf gezählt, denn sie beginnt - um seine Formulierungen zu benutzen -»irgendwo« und gibt dem Hörer erst nachträglich zu erkennen, »wohin die Reise gehen soll.$^{40}$ Zwar ist die Satzeröffnung ımodal uneinheitlich`, doch prägen Melodiebildung und Klauseldisposition im weiteren Verlauf die sneue Tonart klar aus: Klauseln auf a finden sich nur im äolischen Exordium (T. 7) sowie im nachfolgenden, zum Hypojonischen wechselnden Abschnitt (T. 11), während der weitere Verlauf ausschließlich von - vielfach ausgeflohenen - Klauseln auf $c$, der Finalis des neuen Modus, geprägt ist.

Eine Ausnahme unter Byrds Fantasien bildet die bereits erwähnte Voluntary for My Lady Nevell MB XXVIII/61, insofern in ihr kein Moduswechsel stattfindet. Auch darüber hinaus weist sie einen ungewöhnlichen Grad an modaler Regelmäßigkeit auf.

Bereits die nichtimitatorische Introduktion (T. 1-7) weist eindeutig mixolydische Merkmale auf: Der Diskant übersteigt nicht das $d^{2}$, wobei das $h^{1}$ eine bedeutende Rolle spielt. Der mixolydische Charakter des Basses tritt vor allem in der Zurschaustellung des mixolydischen Ambitus G-g gegen Ende der Introduktion deutlich hervor. Insgesamt trägt der Abschnitt Züge einer Intonation des hypomixolydischen Modus.

Der Point des ersten Teils (T. 8 ff.) ist in den beiden Voces regales für den hypomixolydischen Modus charakteristisch: Die traditionelle Quart-Repercussio des Hypomixolydischen, $g-C^{1}$, beziehungsweise $g^{1}-c^{2}$, dominiert im Soggetto trotz seines Anfangs auf der Oberquinte, wobei die Quintspezies des Modus nicht verlassen wird. Die Dominanz der Quart-Repercussio im zweiten Point (T. 17 ff., vgl. Beispiel 3) und dessen Beginn unterhalb der Finalis, mit der Unterquarte $d^{1}$ im Diskant ( $d$ im Tenor) lassen den plagalen Charakter des Hypomixolydischen umso deutlicher hervortreten (Beispiel 3).

Auch in der Melodik des Soggettos im dritten Abschnitt der Fantasie (T. $30 \mathrm{ff}$.) ist die Betonung der hypomixolydischen Repercussa offensichtlich, was wiederum den plagalen Charakter der Tonart hervorhebt. 


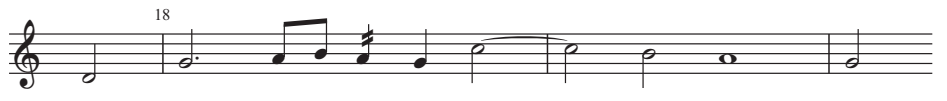

Beispiel 3: William Byrd, Voluntary for My Lady Nevell MB XXVIII/61, zweiter Point, T. 17 ff.

Die Melodik entfaltet sich innerhalb der traditionellen Modusgrenzen: Die Voces regales weisen eindeutig hypomixolydischen Charakter auf, der Bass ist ebenso eindeutig mixolydisch. Nur der Alt weicht teilweise von der modalen Norm ab. ${ }^{41}$

Auch die Klauseldisposition widerspricht den theoretischen Konzepten nicht: Als Klauselebenen fungieren die Finalis ( $g$ ) sowie die plagale und die authentische Repercussa ( $c$, beziehungsweise $d$ ). Klauseln auf $c$, der hypomixolydischen Repercussa, finden sich in großer Dichte in einem frei mehrstimmigen Abschnitt des Werkes (T. 48 ff.). Im dritten Abschnitt der Fantasie (T. 30ff.) steht sogar eine mi-Klausel auf $h$ (T. 33), das zwar die Mitte der Quintspezies des Mixolydischen und Hypomixolydischen darstellt, bekanntlich aber nicht die traditionelle Repercussa des Letzteren ist. Diese Rolle übernimmt das c: Erst Zarlino erklärte die Klausel auf der Oberterz für alle Modi zur Norm. ${ }^{42}$

Alle modal relevanten Merkmale der Komposition weisen auf das Hypomixolydische hin. Mit ihrer den Modus ankündigenden Introduktion, ihrer modal charakteristischen Stimmendisposition und ihrer regelmäßigen Melodiebildung und Klauseldisposition bildet die Voluntary for My Lady Nevell hinsichtlich der Modusbehandlung den Gegenpart der eingangs vorgestellten Hexachordfantasie $U t$, re, mi: Sie entfaltet sich in gelehrter Regelmäßigkeit ganz im Rahmen der traditionellen Moduslehre wie keine andere Fantasie Byrds und kein anderes der »Ladye Nevell « gewidmeten Stücke. ${ }^{43}$

\section{Byrds Fantasien im Spiegel zeitgenössischer Theoriebildung ${ }^{44}$}

Recht häufig in Byrds Fantasien sind Commixtionen von Tonarten mit quint- und terzverwandten Finales, wobei die betreffenden Tonarten entweder identische oder einander diastematisch nahestehende Ambitus aufweisen. Von besonderem Interesse ist in diesem Zusammenhang der Übergang aus dem Hypomixolydischen ins Hypolydische/Lydische in Ut, re, mi: Statt die im Sekundabstand stehenden Modi einfach nebeneinander zu stellen, wird als Zwischentonart das Hypodorische benutzt, das durch den Ton $f^{2}-\operatorname{der}$ im Hypodorischen die traditionelle Repercussa darstellt und im Lydischen und Hypolydischen die Oktave der Finalis ist - eine bedeutende Gemeinsamkeit mit der Zieltonart aufweist. Dass dem Hypodorischen hier tatsächlich eine vermittelnde Funktion zukommt, zeigt sich auch darin, dass das Soggetto der betreffenden Pars zunächt ex $f$

41 Der Alt hat den Ambitus e-c $c^{2}$, wobei der dominierende Umfang von a bis $a^{1}$ reicht, und somit dem mixolydischen Umfang $g-g^{2}$ nahe steht.

42 Vgl. Zarlino 1558/1965, IV, 19 (320).

43 Neighbour $(1978,254)$ ist sich der kompositorischen Strenge dieser Fantasie durchaus bewusst, bringt sie jedoch nicht mit der Modalität in Verbindung.

44 Die beiden nachfolgenden Abschnitte basieren auf einem Teil von Kapitel 5.2 der unveröffentlichten Dissertation des Verfassers (Popovic 2010). 
als hypodorische Oberterz-Repercussa, dann ex $c$ als lydische Oberquint-Repercussa und dann wieder ex $f$, nunmehr aber als lydische Finalis, vorgetragen wird (s. Beispiel 1, T. 28-35). Das konstitutive Element einer Tonart ist zum konstitutiven Element des Tonartwechsels geworden.

In der einzigen Fantasie mit einem dauerhaften Moduswechsel, der Voluntary MB XXVII/27 (s. Beispiel 2), verfährt Byrd anders: Nach der Wende vom Äolischen hin zum Hypojonischen (ab T. 7/T. 53), die sich in den einzelnen Stimmen ungleichzeitig vollzieht, wird der neue Modus konsequent beibehalten, wobei sich die Klauselbildung ganz auf die Clausula primaria des Hypojonischen beschränkt. Mit seinen 23 Klauseln auf $c$ in unmittelbarer Folge mutet dieses Schlussstück der prachtvollen Manuskriptsammlung My Ladye Nevells Booke (1591) in der Tat sehr sschlussbildend an - eine Wirkung, die sich vermutlich nicht erst beim heutigen Hörer einstellt und durchaus in der Intention des Komponisten und/oder Kompilators ${ }^{45}$ der Sammlung gelegen haben könnte. Im Übrigen ist der erste, in My Ladye Nevells Booke nicht zu findende Abschnitt dieser Fantasie (MB XXVII/27, T. 1-46) in tonartlicher Hinsicht auf ähnliche Weise eigentümlich: Alle markanten Klauselbildungen stehen auf dem Ton a, obgleich die Melodik durch das Markieren entscheidender Ecktöne vielfach nahelegt, dass es sich um Hypojonisch handelt (vgl. T. 28-33).

Die häufigen Commixtionen von Tonarten mit quint- und terzverwandten Finales gehen stets mit identischen oder einander diastematisch nahestehenden Tonumfängen einher. Auch der geschilderte Moduswechsel in der Voluntary MB XXVII/27 ist nicht allein ein Übergang von einer Tonart mit Finalis a in eine mit Finalis $C$, sondern auch in eine Zieltonart (Hypojonisch), deren Ambitus $\left(g^{1}-g^{2}\right)$ nur eine Sekunde tiefer liegt als jener der Ausgangstonart ( ̈̈olisch, $a^{2}-a^{2}$ ). Der Moduswechsel findet bei Byrd häufig entweder zwischen zwei Tonarten gleichen oder eine Sekunde entfernten Umfangs statt. Dass die genannten Commixtionen häufig sequenzartig ausgeführt werden, spricht dafür, dass es sich bei der diastematischen Nähe der Tonartumfänge um einen wirklich praxisrelevanten Aspekt des Tonartwechsels handelt.

Bei den meisten Commixtionen - in My Ladye Nevells Booke beispielsweise in allen, außer bei Ut, re, mi - wird aus einer authentisch-modalen Situation (auch wenn es sich insgesamt um ein Moduspaar handelt) in eine plagale sausgewichen Die meisten dieser Moduswechsel finden dabei zwischen Tonarten mit gleichem oder ssekundverwandtem ‘mbitus und quintverwandten Finales statt, wobei diese beiden Aspekte zusammenhängen: Ein authentischer Modus hat durch die Teilung der Oktave in zwei Spezies den gleichen Umfang wie die plagale Tonart, deren Finalis seine Oberquarte ist (z. B. Jonisch-Hypolydisch), und ein plagaler Modus hat einen eine Sekunde höheren Ambitus als die authentische Tonart, auf deren Oberquint die Finalis der Ersteren liegt (z. B. Hypomixolydisch-Jonisch).

Diese Überlegungen wären geeignet, eine wörtliche Lesart der bereits vorgestellten These Morleys zu stützen, wonach ein Moduswechsel nur zwischen einem authenti-

45 Als Kompilator dürfte der im Manuskript selbst unterzeichnete John Baldwin gelten, ein renommierter zeitgenössischer Notenschreiber und Musiker. Zu Baldwin s. etwa Mateer 2004 und die daselbst angeführte Literatur; zu Baldwins Arbeit an My Ladye Nevells Booke s. Gaskin 1992 sowie Popovic 2010. 
schen und einem plagalen Modus stattfinden darf. ${ }^{46}$ Obwohl Morleys eigene Beispiele nahe legen, er habe eigentlich die Mixtio - den Übergang in die Tonart gleicher Finalis und unterschiedlichen Umfangs - gutheißen und Commixtio - den Übergang in eine völlig fremde Tonart - verbieten wollen ${ }^{47}$, zeigen die Analysen, dass in der Kompositionstechnik William Byrds neben der Mixtio eben auch die Commixtio zwischen einem plagalen und einem authentischen Modus möglich war.

Ähnlich wie die Disposition der modalen Gesamtkonzeption der Werke ist auch Byrds Kompositionspraxis im Einzelnen angelegt: Elemente der traditionellen, regulären Modusbehandlung überschneiden sich mit unkonventionellen Aspekten. Wie an den obigen drei Beispielen demonstriert, bewegen sich - innerhalb der jeweils modal definierbaren Abschnitte - Byrds Melodiebildung sowie die Stimmendisposition im Rahmen der modalen Normen.

Obwohl die Disposition der Klauseln innerhalb von modal bestimmbaren Abschnitten zum großen Teil der Norm entspricht, sind außerhalb von Commixtionen zuweilen Clausulae peregrinae vorzufinden. ${ }^{48}$ Es ist kaum möglich, eine Regel zu formulieren, die Byrds Gebrauch dieser Klausel (weg)erklären würde: Die Argumentation kann nicht, wie im Fall der Vokalmusik, in Richtung hermeneutischer Spekulationen gelenkt werden, die die sfremdartigen Klauselstufen als Textausdeutung rechtfertigen würden. ${ }^{49}$ Generalisierend lasst sich feststellen, dass die Klausel auf der Oberterz, die in den traditionellen Moduslehren zu den regulären Klauseln gehört, in Byrds Fantasien verhältnismäßig selten zu finden ist. ${ }^{50}$ Ihren Platz als Tertiaria nimmt zumeist die Klausel auf der Oberquarte ein, unabhängig davon, ob die Tonart dural oder mollar ist. ${ }^{51}$ In zwei der genannten Fantasien - Ut, re, mi und Voluntary for My Lady Nevell - wurde mit dem Hypomixolydischen eine Tonart gewählt, in der die Oberterz als Clavis clausularum traditionell gemieden wurde. Gerade aber in einer dieser Kompositionen, der sonst überaus konventionellen Voluntary for My Lady Nevell, findet sich eine Klausel auf der Oberterz $h$. Dies ist vermutlich kein Zufall: Diese sgelehrte Voluntary ist, wie bereits ausgeführt, von einer eigentümlichen und unter Byrds Fantasien einzigartigen modalen Regelmäßigkeit, die möglicherweise auf große italienische Vorbilder hinweist. Und es war gerade Gioseffo Zarlino, der die Klauseldisposition sämtlicher Modi dem theoretischen Schema I-V-III unterworfen hatte $^{52}$, so dass auch in den Modi mit der Finalis $G$ die sonst gemiedene Oberterz als reguläre Klauselstufe galt. Nach Zarlinos Theorie, auf die sich etwa Thomas Morley in

46 Vgl. Morley 1597/1937, „Annotations upon the third part", o. S.

47 Positivbeispiele Morleys sind die Kombinationen: I. und II., III. und IV. Modus »\&c.«: Morley 1597 / 1937, „Annotations upon the third Part«, o. S.

48 Die innerhalb von Commixtionen stattfindenden Klauseln werden in Bezug auf den vorläufigen Modus, nicht in Bezug auf die Haupttonart bezeichnet.

49 Ein Ansatz, der insbesondere für Bernhard Meier charakteristisch war (1974, vgl. hierzu Dahlhaus' Kritik in Dahlhaus 2006a sowie 1976).

50 Für eine detaillierte Ausführung dieses Sachverhalts vgl. Popovic 2010.

51 Es sei in diesem Kontext daran erinnert, dass der zeitgenössische englische Theoretiker Thomas Campion die Klauseln auf der Obersekunde und der Oberquarte nur in den duralen Modi zu regulären Klauseln gezählt hat (vgl. 1967a, 214f.).

52 Vgl. Zarlino 1558/1965, IV, 19 (320). 
seinen »Annotations « ausdrücklich beruft ${ }^{53}$, wurde also das $h / H$ als Clavis clausularum im Hypomixolydischen und Mixolydischen des Cantus durus als akzeptabel betrachtet.

Diese Anlehnung an das berühmte italienische Vorbild und die modal regelmäßige Anlage der Voluntary for My Lady Nevell weisen möglicherweise einen Bezug zur Widmungsträgerin Lady Elizabeth Neville auf. Wie bereits erwähnt, entstammte sie einem Umfeld von Gelehrten und war selbst Patronin von Kunst, Wissenschaft und Bildung. Eine ausgesprochen ıgelehrte Fantasie erscheint ihr als Widmungskomposition in besonderer Weise angemessen. Andererseits könnten die Eigenschaften dieser Fantasie auch dazu verleiten, sie als ein sfrühes` Werk zu betrachten. Freilich hieße dies, von einer unilinearen sstilistischen` Entwicklung des Komponisten auszugehen, was jedoch zu simpel anmutet, um der Komplexität der kompositorischen Wirklichkeit Rechnung zu tragen. ${ }^{54}$ Zudem wäre zu fragen, warum gerade das vermeintliche `Frühwerkı und nicht eine der anderen, skomplexeren Fantasien aus My Ladye Nevells Booke expressis verbis Lady Elizabeth Neville gewidmet wurde.

Schließlich soll der Blick auch auf den bisher nicht angesprochenen Aspekt der Exordialimitation gerichtet werden. Maßgeblich von Zarlino formuliert wurde ${ }^{55}$ die im 16 . und 17. Jahrhundert zunehmend praxisrelevante ${ }^{56}$ Regel, wonach die Imitationsmotive entweder auf der Finalis (und ihrer Oberoktave) oder auf der Oberquinte (bzw. Unterquarte) des Modus beginnen müssen. In drei seiner Fantasien verwendet Byrd jedoch nicht dieses ımodernisierte`, vereinfachte Schema der Imitationsebenen, sondern imitiert auch auf der Oberquarte oder Unterquinte des Modus. Diese ältere Imitationsform ist etwa im Exordium der analysierten Hexachord-Fantasie $U t$, re, mi sowie in den Exordien der Lesson of Voluntary MB XXVII/26 und der Fancy MB XXVIII/46 zu finden. Dabei ist bemerkenswert, wie Byrd im hypomixolydischen Modus verfährt, in dem nach alter Praxis die Imitation auf der Unterquinte als korrekt erachtet wurde. ${ }^{57}$ In der oben beschriebenen hypomixolydischen Hexachord-Fantasie Ut, re, mi benutzt er diese alte Imitationsweise, während im Exordium der Voluntary for My Lady Nevell gerade die von Zarlino gebotene Oberquint-Imitation ex $d$ stattfindet. Womöglich kann auch dieser Umstand, neben allen anderen genannten Merkmalen dieser Komposition, als ein weiterer Hinweis auf den gelehrten, wahrscheinlich an italienischen Vorbildern geschulten Geschmack der Lady Neville verstanden werden.

Neighbour betont in seiner vorwiegend den Aspekten der formalen Gliederung und der Satztechnik sowie der Gattungsgeschichte gewidmeten Studie über die Instrumentalmusik Byrds die Individualität der kompositorischen Konzepte Byrds. ${ }^{58}$ Diese Individualität betrifft auch die Modusbehandlung. Den Fantasien Byrds liegen nicht nur unterschiedliche Kombinationen von Tonarten zugrunde, sondern auch unterschiedliche Arten, diese Kombinationen zu verwirklichen, sowie eine andere Behandlungsart des

53 Vgl. Morley 1597/1937, »The Annotations upon the third part« sowie Literaturverzeichnis (o. S.).

54 Die Problematik des musikalisches ıStilbegriffs` ist zu komplex, um im Rahmen dieses Beitrags erörtert zu werden. Ein Überblick über die Thematik ist etwa in Seidel/Leisinger 1998 zu finden.

55 Vgl. Zarlino 1558/1965, III, 28.

56 Vgl. Meier 1992a, 96-104.

57 Vgl. ebd. 
Modus verus. Die Hexachordfantasie Ut, re, mi, folgt der Idee der Hexachord-SoggettoDurchführung auf verschiedenen Ebenen, beinhaltet Klauseln auf allen sechs Stufen des Hexachords und ist durch komplexe Commixtionen gekennzeichnet. Am anderen Ende des Spektrums der Tonartbehandlung steht die streng modale und `Zarlino-konforme` Voluntary for My Lady Nevell. In der Voluntary MB XXVII/27 schließlich findet ein dauerhafter Moduswechsel statt, und es werden - ebenfalls einzigartig in Byrds Fantasien - zur Schlussbildung ausschließlich die Clausulae primariae der beiden beteiligten Modi verwendet. Die anderen Fantasien Byrds weisen wiederum andere Tonartenkonzepte auf, die zu behandeln den Rahmen dieses Beitrags sprengen würde. ${ }^{59}$

Die Modusbehandlung in den Fantasien Byrds unterscheidet sich nicht so sehr durch die Radikalität der `Ausnahmefälle` von jener der bedeutenden Komponisten des europäischen Festlands, als vielmehr durch die Vielfalt und Dichte dieser >Ausnahmefälle «: Die Modi werden in diesen instrumental-polyphonen Kompositionen Byrds nicht izersetzts oder saufgehoben`, sondern erweitert, modifiziert, miteinander kombiniert und in konzentrierter Form präsentiert. Und die harmonische Tonalität hat sich - wie wir spätestens seit Dahlhaus' Untersuchungen über die Entstehung der harmonischen Tonalität ${ }^{60}$ wissen - eben auf dem Wege der Erweiterung, Modifikation, Kombination und Konzentration von Elementen und Gestaltungsprinzipien modaler Tonalität, nicht auf dem Weg ihrer Zersetzung entfaltet. Die Tonartlichkeit der hier analysierten Kompositionen Byrds ist bereits bedeutend erweitert im Sinne der polymodalen Gesamtkonzeptionen und modifiziert im Sinne Byrds eigener Klauseldispositionsschemata. Sie ist aber nicht ausschließlich konzentriert auf Merkmale, die man anachronistisch als sharmonisch bezeichnen könnte: Nach wie vor ist die Melodik konstitutiv für den Prozess des Tonartwechsels, und die Disposition der Schlussbildungen ist keineswegs nur durch QuintQuart-Verhältnisse oder die Unterscheidung zwischen mollaren und duralen Modi geprägt. Gerade deshalb stellen die instrumental-polyphonen Kompositionen Byrds einen wichtigen Orientierungspunkt im Prozess der `Entstehung der harmonischen Tonalitätı dar.

\section{`Byrdsche` oder senglische` Tonartbehandlung? Ein kulturhistorischer Ausblick}

War aber William Byrd als das komponierende Subjekt in diesem Prozess die entscheidende Instanz, der die Modifikation und Umfunktionierung der alten Tonarten zuzuschreiben ist? Oder haben entsprechende Verfahrensweisen ihre Verankerung primär im epochenbezogenen und/oder regionalen Musikdenken? Die noch vor Byrds Geburt vollzogene Trennung Englands von dem katholisch geprägten europäischen Festland hatte auch die Trennung von den Kulturgütern der Römisch-katholischen Kirche zur Folge. Diese wiederum führte nicht nur zur Entwicklung neuer Musikgattungen ${ }^{61}$, sondern hatte

58 Vgl. Neighbour 1978, passim.

59 Vgl. dazu Popovic 2010, Kapitel 5.2 sowie Anhang zu Teil 5.

60 Dahlhaus 2001a.

61 Vgl. hierzu, in bezug auf Musik für Tasteninstrumente, insbesondere Edler 1997, 355. Auch Seidel entgeht die soziale Bedingtheit der Entstehung von Byrds Fantasien nicht (vgl. den kurzen, aber treffenden Hinweis in Seidel 1987, 111). 
auch Auswirkungen auf die Behandlung der alten Tonarten, die ja traditionell mit dem kirchlichen Raum verbunden waren. Zwar dürfte Byrd - wie man etwa nach den bei Morley zitierten Komponisten vermuten darf - genug Gelegenheit gehabt haben, sich mit dem sakralen Musikgut des kontinentalen Europas und den entsprechenden musiktheoretischen Traktaten auseinander zu setzen. Auch mag die kontinentaleuropäische Musiktheorie über seinen mutmaßlichen Lehrer Thomas Tallis tradiert worden sein. ${ }^{62}$ Jedoch konnte Byrd nach dem Tod der römisch-katholischen Königin Mary und des Erzbischofs Reginald Pole sowie der darauf folgenden Thronbesteigung Elizabeths I. (1558) nicht in permanentem Kontakt mit der Kultur der römischen Kirche bleiben. Es war ihm nicht möglich, an Gottesdiensten in großen römisch-katholischen Kathedralen und Abteien teilzunehmen und sich konstant und aktiv mit den Produkten ihrer Musikkultur zu befassen. Sein Katholizismus war vielmehr gesetzeswidrig und durfte nur im Verborgenen praktiziert werden. ${ }^{63}$ Gleichzeitig schwanden im elisabethanischen England die Choralkultur und die Kultur des improvisierten Diskantierens und Psalmodierens ${ }^{64}$, während die Literalisierung der Instrumentalmusik fortschritt. ${ }^{65}$ Selbstverständlich schloss der Protestantismus nicht automatisch die Präsenz der Moduslehre aus, die ja auch im protestantischen Kontinentaleuropa weit verbreitet war. Es dürfte nicht zuletzt die Insularität Englands dazu beigetragen haben, dass die Moduslehre im England des 16. Jahrhunderts nicht in dem Maße und in der gleichen Weise rezipiert und fortgeschrieben wurde, wie auf dem europäischen Festland. Die substantiellen Veränderungen der Tonartenkonzepte innerhalb der gesamten Kompositionspraxis erscheinen vor diesem Hintergrund verständlich.

John Harley, der seine Untersuchungen der Moduspraxis Byrds hauptsächlich auf dessen Vokalkompositionen beschränkt, behandelt den Tonartwechsel als einen wichtigen Aspekt der Byrdschen Kompositionen. ${ }^{66}$ Auch in Bernhard Meiers Standardwerk über die Modusbehandlung in der Vokalmusik finden Beispiele Byrds, die charakteristische Tonartwechsel aufweisen, Erwähnung. ${ }^{67}$ In der polyphonen Musik für Tasteninstrumente Byrds äußert sich diese Tendenz aber als konstitutiver Aspekt der Kompositionspraxis, so dass das auf dem Kontinent nach wie vor geltende Prinzip der `Tonarteinhaltung bei Byrd geradezu zur Ausnahme wird.

Harley führt den möglicherweise frühen, modal streng regelkonformen liturgischen Satz Byrds Alleluia. Confitemini Domino als Beleg dafür an, dass Byrd in seiner Jugend mit der kontinentalen Moduslehre in Kontakt gekommen sei und dass sie Einfluss auf seine Kompositionstechnik ausgeübt habe.$^{68}$ Gleichwohl scheint es unangemessen, von hier

62 Tallis war im Besitz einiger musiktheoretischer Traktate, die er anscheinend aus der 1540 aufgelösten Abtei Waltham gerettet hatte; vgl. hierzu Harley 2005, 3.

63 Die Literatur zu Byrds Katholizismus ist zu umfangreich, um hier systematisch vorgestellt zu werden; es seien im biographischen Sinne insbesondere Harley 1997, 68f., 126-131 sowie Mateer 1996 hervorgehoben. Vgl. in Bezug auf Byrds Schaffen Kerman 1981, Monson 1997, sowie McCarthy 2007.

64 Vgl. hierzu insbesondere die Autobiographie Thomas Whythornes (1961, 203 f.).

65 Zum Schwinden der vokalen Improvisationskultur im elisabethanischen England insbesondere im kirchlichen Raum vgl. etwa Flynn 1993, $277 \mathrm{f}$.

66 Vgl. Harley 2005, 84-87.

67 Vgl. Meier 1974, 294, 306.

68 Vgl. Harley 2005, 2. 
aus eine `Entwicklung`Byrds hin zu einer `Befreiung` von modalen `Zwängen` nach dem Modell des spätneuzeitlichen Fortschrittdenkens zu konstruieren. Es gibt nicht genügend Hinweise darauf, dass die Relativierung der traditionellen Tonarten das Produkt einer rein subjektiven Entwicklung Byrds ist, während andererseits verschiedene Hinweise - nicht zuletzt die Vagheit der Ausführungen Morleys zu den Modi - dafür sprechen, dass die Moduslehre zu Byrds Zeiten im englischen Musikdenken generell keine zentrale Rolle mehr gespielt hat: Sie war bekannt, galt aber nicht mehr als verbindlich.

Die Fantasien anderer Komponisten des 16. Jahrhunderts und der frühen Stuart-Epoche (ab 1603) sind ebenfalls relativ weit entfernt von der modalen Tradition. Ein umfassender Vergleich dieser Kompositionen mit denjenigen Byrds kann hier nicht geleistet werden, doch vermag ein Blick auf ihre Tonartbehandlung voreiligen Schlüssen hinsichtlich Byrds Einzigartigkeit vorzubeugen. Schon in dem früheren Mulliner Book ${ }^{69}$ sind Anzeichen von Tonartwechseln in polyphonen Werken vorzufinden: Richard Farrants (um 1525-1580) Felix namque aus dieser Sammlung liefert ein gutes Beispiel (MB I/19, etwa T. 75-95), ebenso Thomas Tallis' (um 1505-1585) Felix namque-Sätze MB LXVI/4 und 5. Auch Thomas Morley (1557/8-1602), der einer jüngeren Generation angehörte, folgte nicht immer seinem eigenen Rat, Moduswechsel in Fantasien zu vermeiden ${ }^{70}$, sondern bevorzugte, wie Byrd, einen freien tonartlichen Verlauf: Bernhard Meier führt gerade Morleys Fantasia aus Fitzwilliam Virginal Booke (Nr. 57) als ein Beispiel für den ımodal uneinheitlichen Verlauf in der Instrumentalmusik des 16. Jahrhunderts an. ${ }^{71}$ Dabei mögen mikrosoziologische Gründe eine Rolle gespielt haben, da Morley William Byrd nahe stand und auch betonte, sein Schüler gewesen zu sein. ${ }^{72}$

In der Fancy for Viols (MB V/33) $)^{73}$ des Byrd-Schülers Thomas Tomkins (1572-1656) lassen sich ähnlich angelegte Tonartveränderungen beobachten wie bei William Byrd (MB V/33, T. 6-26), die jedoch stärker chromatisch profiliert sind. Allerdings etablieren sich sowohl hier als auch in anderen gattungsverwandten Kompositionen Tomkins ${ }^{\prime 74}$ die $: Z$ wischentonarten nicht längerfristig. Eine entsprechende Tonartbehandlung findet sich in der Musik für Tasteninstrumente unter anderem in Alfonso Ferraboscos Fancy MB LXVI/31 (T. 35-42). Die drei überlieferten Fantasien (MB LXXVI/11-13) von Peter Philips (1560/1-1628), der wahrscheinlich Byrds Schüler war ${ }^{75}$, zeigen dagegen keine relevanten Ausweichungen. ${ }^{76}$

Ein Vergleich zwischen William Byrd (1540-1623) einerseits sowie John Bull (1562/3-1628) und Orlando Gibbons (1583-1625) anderseits - beide Angehörige einer jüngeren Generation und nicht als Byrd-Schüler überliefert -, zeigt, dass vor allem die

69 Ausgabe: MB I. Das Manuskript ist nicht exakt datierbar.

$70 \mathrm{Vgl}$. Morley 1597/1937, 147.

71 Meier 1992a, 156

72 Vgl. Morley 1597/1937, Widmung (o. S.).

73 Auch in Paris MS 1122, einer Sammlung für Tasteninstrumente, enthalten.

74 Vgl. etwa seine Fancy MB V/23, Voluntary MB V/24, die kurze Voluntary MB V/30 sowie Substantial Verse $\mathrm{MB} \mathrm{V/31.}$

75 Vgl. David Smiths Einleitung in MB LXXVI, XVII.

76 David Smith vermutet, dass die drei Fantasien Philips' lediglich Intavolierungen von Vokalwerken darstellen (vgl. ebd., XIX). 
Verfahren, die traditionellen Tonarten zu relativieren, individuell geprägt sind. So kommen in Bulls Fantasien ${ }^{77}$ zwar Wendungen vor, die an Byrds Tonartwechsel erinnern, doch gibt es deutliche Unterschiede in der Detailarbeit. In der dorisch-hypodorischen Fantasie aus Prelude and Fantasia MB XIV/1 findet ein Tonartwechsel in den Bereich des Jonisch-Hypojonischen (T. 85) statt. Dieser ist - ähnlich wie in manchen Kompositionen Byrds - als Sequenzierung des vorausgegangenen Abschnittes (T. 80-83) zu verstehen. Die Rückkehr in die ursprüngliche Tonart erfolgt dabei wiederum über eine Sequenz (T. $100 \mathrm{f}$.). Häufig bei Bull sind aber auch Wendungen, die nicht zur Etablierung einer neuen Tonart, sondern zu kurzzeitigen, modal nicht genau bestimmbaren und meistens im virtuosen Kontext stattfindenden `Ausweichungen` führen. Ein Beispiel dafür findet sich in der bereits zitierten Fantasia MB XIV/1: In einem dorisch-hypodorischen Gesamtkontext werden die Bereiche des Lydischen/Hypolydischen (T. 33, 35) und TransponiertDorischen (T. 39f.) sequenzartig angedeutet; die weitere Entfaltung der Komposition erfolgt in der Ausgangstonart (ab T. 41). Eine vergleichbare Tonartbehandlung ist in anderen Fantasien Bulls ebenfalls zu finden. Interessant sind in diesem Zusammenhang insbesondere die mit Modusbezeichnungen versehenen Fantasien MB XIV/2 (Prelude and Fantasia in the Eighth Mode) und MB XIV/17 (Fantasia in the Fifth Mode) John Bulls: Obgleich sich in ihnen keine länger andauernden Tonartwechsel finden, verhindern die Chromatik einerseits und der virtuose Instrumentalsatz andererseits über weite Strecken eine deutliche Manifestation der Tonarten. ${ }^{78}$

Auch Orlando Gibbons' Fantasien ${ }^{79}$ können weder als modal regelmäßig beschrieben werden noch sind für sie länger währende Ausweichungen jener Art charakteristisch, wie sie in den Fantasien Byrds die Regel darstellen. Bei Gibbons wird die Strenge der Melodiebildung durch einen spezifisch-instrumentalen Satz relativiert, während zugleich die Tendenz erkennbar ist, den Tonvorrat und die Klauseln ein und derselben Tonart - mit kürzeren Ausweichungen - immer wieder einzusetzen. Hinsichtlich der Tonartbehandlung scheint der zentrale Unterschied zwischen Byrd einerseits und Bull und Gibbons andererseits darin zu bestehen, dass Ersterer die alten Tonarten teils bewahrt, teils die mit Ihnen verbundenen Verfahren und Charakteristika modifiziert, um sie auf einer übergeordneten Ebene wieder miteinander zu kombinieren, während die beiden anderen Komponisten häufige Ausweichungen kürzerer Dauer bevorzugen und so das Moduskonzept im Detail relativieren.

Byrds Zeitgenosse Henry Peacham beschrieb den römisch-katholischen Komponisten als »naturally disposed to gravity and piety«. ${ }^{80}$ Womöglich ist die geschilderte Behandlung von Tonarten in seinen Fantasien auch mit dieser >Disposition « in Verbindung zu bringen: Trotz der generellen Tendenz, die Bedeutung der traditionellen Tonartenkonzepte zu relativieren, ist bei Byrd im kompositionstechnischen Detail mehr von den

77 MB XIV/1-19.

78 Die beiden zitierten Kompositionen Bulls sind dabei in einem niederländischen Manuskript überliefert und die Moduszuschreibung mag auf den Kompilator Messaus zurückgehen, der als Kirchenmusiker in Antwerpen arbeitete, wo auch Bull tätig gewesen war. Vgl. hierzu MB XIV, 159.

$79 \mathrm{MB} X X / 1-14$.

80 Peacham 1962, 112; mit Originalorthographie zitiert in Harley 1997, 166. 
alten Tonarten der klassischen Vokalpolyphonie geblieben als bei Bull und Gibbons. Die gesellschaftlichen Umbrüche des Tudor-Zeitalters beeinflussten den allgemeinen Stellenwert der alten Tonarten im elisabethanischen England; die Tonartbehandlung im Einzelnen war aber offenbar durch mikrosoziologische und subjektive Faktoren geprägt.

\section{Zitierte musikalische Quellen}

Fitzwilliam Virginal Book = John Alexander Fuller Maitland, William Barclay Squire (Hg.), The Fitzwilliam Virginal Book, London: Dover Publications 1963 [Leipzig 1899], 2. Bde. $\mathrm{MB}=$ Anthony Lewes u.a. (Hg.), Musica Britannica. A National Collection of Music, London: Steiner \& Bell 1951.

MB I = Denis Stevens (Hg.), The Mulliner Book, London: Steiner \& Bell 1973.

MB V = Stephen D. Tuttle (Hg.), Thomas Tomkins. Keyboard Music, London: Steiner \& Bell 1965.

MB XIV = John Steele, Francis Cameron (Hg.), John Bull. Keyboard Music I, London: Steiner \& Bell 1960.

MB XIX = Thurston Dart (Hg.), John Bull. Keyboard Music II, London: Steiner \& Bell 1963. MB XX = Gerald Hendrie (Hg.), Orlando Gibbons. Keyboard Music, London: Steiner \& Bell 1962.

MBXXVII = Alan Brown (Hg.), William Byrd. Keyboard Musicl, London: Steiner \& Bell 1969. MBXXVIII=Alan Brown(Hg.), William Byrd. KeyboardMusic II, London:Steiner \& Bell 1971. MB LXVI = John Caldwell (Hg.), Tudor Keyboard Music c. 1520-1580, London: Steiner \& Bell 1995.

MB LXXVI = David J. Smith (Hg.), Peter Philips. Complete Keyboard Music, London: Steiner \& Bell 1999.

My Ladye Nevells Booke = Hilda Andrews (Hg.), My Ladye Nevells Booke of Virginal Music by William Byrd, London u. a.: Curwen 1926.

Paris MS 1122 = Paris, La Bibliothéque du Conservatoire, Manuscrit Réserve 1122.

Parthenia = Parthenia or the Maydenhead of the first musicke that ever was printed for the virginalls / comp. by 3 famous masters William Byrd, Dr John Bull \& Orlando Gibbons, London: William Hole 1612/13, Reprint London: Chiswick Press 1942 [= The Harrow Replicas 3].

\section{Lexika und Handbücher}

DNB = Matthew, H.C. G.; Harrison, Brian (Hg.), Oxford Dictionary of National Biography, 60 Bde., Index of Contributors, Oxford u. a.: Oxford University Press 2004.

MGG2 = Die Musik in Geschichte und Gegenwart. Allgemeine Enzyklopädie der Musik. 2. neubearb. Auflage, hg. von Ludwig Finscher, Kassel u.a.: Bärenreiter u.a. 1994-2008. 


\section{Literatur}

Apel, Willy (1972), The History of Keyboard Music to 1700, Bloomington: Indiana University Press.

Bourdieu, Pierre (1992), Die verborgenen Mechanismen der Macht, hg. von Margareta Steinrücke (= Pierre Bourdieu, Schriften zu Politik \& Kultur, hg. von Margareta Steinrücke, Bd. 1), Hamburg: VSA-Verlag.

Boyd, Morrison Comegys (1962), Elizabethan Music and Musical Criticism, Philadelphia: University of Pennsylvania Press.

Brown, Alan und Richard Turbet (Hg.) (1992), Byrd Studies, Cambridge: Cambridge University Press.

Butler, Charles (1636), The Principles of Musik in Singing and Setting, London: J. Haviland, Reprint New York: Da Capo Press Inc. 1970.

Campion, Thomas (1967), Campion's Works, hg. von Percival Vivian, Oxford: Clarendon Press.

(1967a), »A new Way of Making fowre parts in Counter-point, by a most familiar, and infallible Rule«, in: Campion 1967, 189-226.

Dahlhaus, Carl (1976), „Zur Tonartenlehre des 16. Jahrhunderts. Eine Duplik», Die Musikforschung 29, 300-303.

- (2001), Alte Musik. Musiktheorie bis zum 17. Jahrhundert - 18. Jahrhundert (= Gesammelte Schriften, Bd. 3, hg. von Hermann Danuser u. a.), Laaber: Laaber.

(2001a), »Untersuchungen über die Entstehung der harmonischen Tonalität», in: Dahlhaus 2001, 11-307.

— (2001b), »Die Termini Dur und Moll«, in: Dahlhaus 2001, 312-328.

— (2006), Rezensionen (= Carl Dahlhaus Gesammelte Schriften, Bd. 9, hg. von Hermann Danuser u.a.), Laaber: Laaber.

(2006a), »Bernhard Meier, Die Tonarten der klassischen Vokalpolyphonie. Nach den Quellen dargestellt, Utrecht 1974«, Rezension, in: Dahlhaus 2006, 316-319.

Dalmonte, Rossana und Mario Baroni (1992) (Hg.), Secondo Convegno Europeo di Analisi Musicale, Trento: CENFOR.

Danuser, Hermann (1975), Musikalische Prosa, Regensburg: Bosse.

_ (1995), Artikel »Gattung«, in: MGG2, Sachteil, Bd. 3, Sp. 1042-1070.

_ (1997), Artikel »Musikalische Prosa», in: MGG2, Sachteil, Bd. 6, Sp. 857-866.

Desroches, Dennis (2006), Francis Bacon and the Limits of Scientific Knowledge, London: Continuum.

Edler, Arnfried (1997), Gattungen der Musik für Tasteninstrumente, Teil 1: Von den Anfängen bis 1750 (= Handbuch der musikalischen Gattungen 7/1), Laaber: Laaber.

Flynn, Jane E. (1993), A Reconsideration of the Mulliner Book (British Library Add. MS 30513): Music Education in Sixteenth-Century England, PhD Diss., Duke University 1993, University Microfilms 9416896. 
Foley, Christopher (2005), My Ladye Nevells Booke. The Most Important and Famous Keyboard Manuscript Surviving in Private Hands from $16^{\text {th }}$ Century England, Containing 42 Works by William Byrd, "the Greatest Composer" of the Elizabethan Age, MS (verfasst für Lord Abergavenny).

Gaskin, Hilary (1992): »Baldwin and the Nevell Hand«, in: Brown/Turbet 1992, 159-173. Harley, John (1997), William Byrd. Gentleman of the Chapel Royal, Aldershot: Ahsgate.

— (2005), William Byrd's Modal Practice, Aldershot: Ashgate.

_ (2005a): » My Ladye Nevell Revealed", in: Music \& Letters 86, 1-13 (mit Anhang).

Hauge, Peter (1997), English Music Theory c. 1590-c. 1690: The Modal Systems, Changing Concepts, and the Development of New Classification System, PhD Diss., City University London.

Hill, John W. (Hg.) (1980), Studies in Musicology in Honor of Otto E. Albrecht, Kassel u. a.: Bärenreiter u.a.

Horn, Wolfgang (2005), , Est modus in rebus ... Gioseffo Zarlinos Musiktheorie und Kompositionslehre und das 'Tonarten «-Problem in der Musikwissenschaft, Habilitationsschrift, Hochschule für Musik und Theater Hannover (Druck i. V.).

Judd, Cristle Collins (Hg.) (1998), Tonal Structures in Early Music, New York u. a.: Garland. Kerman, Joseph (1981), The Masses and Motets of William Byrd (= The Music of William Byrd 1), London u.a.: Faber u.a.

Klotz, Martin (2005), Instrumentale Konzeptionen in der Virginalmusik von William Byrd, Tutzing: Schneider.

Mateer, David (1996), „William Byrd, John Petre and Oxford, Bodleian MS Mus. Sch. E. 423: An Index and Commentary«, in: Royal Musical Association Research Chronicle 29, 21-46.

— (2004), Artikel »John Baldwin (d. 1615)«, in: DNB, Bd. 3, $452 \mathrm{f}$.

McCarthy, Kerry (2007), Liturgy and Contemplation in Byrd's Gradualia, New York u.a.: Routledge.

Meier, Bernhard (1974), Die Tonarten der klassischen Vokalpolyphonie, Utrecht: Oosthoek, Scheltema \& Holkema.

— (1977), „Die Modi der Toccaten Claudio Merulos (Rom 1598 und 1604)«, in: Archiv für Musikwissenschaft 34 (1977), 180-198.

_ (1992a), Alte Tonarten. Dargestellt an der Instrumentalmusik des 16. und 17. Jahrhunderts (= Bärenreiter Studienbücher Musik 3), Kassel u. a.: Bärenreiter.

— (1992b), »Auf der Grenze von modalem und dur-moll-tonalem System«, in: Basler Jahrbuch für historische Musikpraxis 16, 53-69.

Monson, Craig (1997), »Byrd, the Catholics, and the Motet. The Hearing Reopened«, in: Pesce 1997, 348-374.

Morley, Thomas (1597), A Plaine and Easie Introduction to Practicall Musicke, London: Peter Short, Reprint London: Milford 1937 (= Shakespeare Association Facsimiles 14).

Neighbour, Oliver (1978), The Consort and Keyboard Music of William Byrd (= The Music of William Byrd 3), London u. a.: Faber u.a. 
Palisca, Claude V. (1985), Humanism in Italian Renaissance Musical Thought, New Haven u. a.: Yale University Press.

(1994), Studies in the History of Italian Music and Music Theory, Oxford: Clarendon Press.

Peacham, Henry (1962), The Complete Gentleman, Ithaca: Cornell University Press.

Pesce, Dolores (Hg.) (1997), Hearing the Motet. Essays on the Motet of the Middle Ages and Renaissance, New York u. a.: Oxford University Press.

Popovic, Tihomir (2010), Mäzene - Manuskripte - Modi. Musiktheoretische und musikhistorische Untersuchungen zu >My Ladye Nevells Booker, Phil. Diss., Humboldt-Universität zu Berlin (Druck i. V.).

Powers, Harold (1981), »Tonal Types and Modal Categories in Renaissance Polyphony«, Journal of the American Musicological Society 24 (1981), 428-470.

(1989), »Modal Representation in Polyphonic Offertories«, in: Early Music History 2, 47-86.

(1992a), »Is Mode Real? Pietro Aron, the Octenary System, and Polyphony«, in: Basler Jahrbuch für historische Musikpraxis 16, 9-52.

(1992b), »Modality as a European Cultural Construct«, in: Dalmonte/Baroni 1992, 207-219.

— (1998), »From Psalmody to Tonality«, in: Judd 1998, 275-340.

Praetorius, Michael (1619), Syntagma musicum III: Termini musici, Wolfenbüttel 1619, Reprint Kassel u.a.: Bärenreiter 1978.

Seidel, Wilhelm (1987), Werk und Werkbegriff in der Musikgeschichte (= Erträge der Forschung 246), Darmstadt: Wissenschaftliche Buchgesellschaft.

Seidel, Wilhelm und Ulrich Leisinger (1998), Artikel »Stil«, in: MGG2, Sachteil, Bd. 8, Sp. 1740-1759.

Smith, Richard Michael (Hg.) (1972): The Sir Nicholas Bacon Collection. Sources on English Society 1250-1700. A Catalogue of an Exhibition at the Joseph Regenstein Library of the University of Chicago 1972, Chicago: University of Chicago Library.

Solomon, Julie Robin und Catherine Gimelli Martin (Hg.) (2005), Francis Bacon and the Refiguring of Early Modern Thought. Essays to Commemorate The Advancement of Learning (1605-2005), Aldershot: Ashgate.

Titler, Robert (1976), Nicholas Bacon: The Making of a Tudor Statesman, London: Cape. (2004), Artikel »Bacon, Sir Nicholas«, in: DNB, Bd. 3, 165-170.

Whythorne, Thomas (1961), The Autobiography, hg. von James M. Osborn, Oxford: Clarendon Press.

Zarlino, Gioseffo (1558), Le Istitutioni harmoniche, Venedig: Francesco Senese, Reprint New York: Broude 1965.

Zimmermann, Franklin B. (1980), „Air, a Catchword for New Concepts in SeventeenthCentury English Music Theory«, in: Hill 1980, 142-157. 